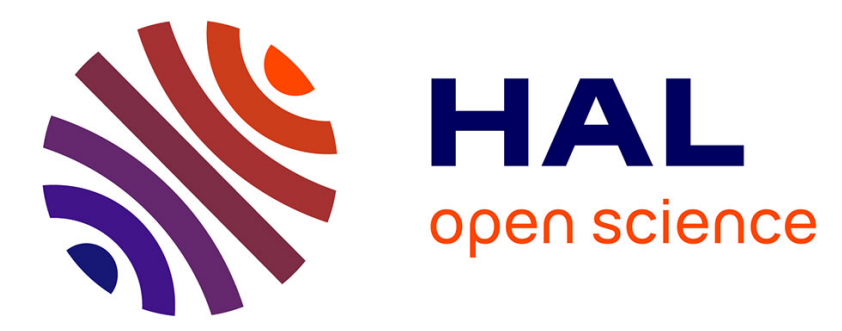

\title{
Fluctuations in inertial dense homogeneous suspensions
} Elise Alméras, Olivier Masbernat, Frédéric Risso, Rodney O. Fox

\section{To cite this version:}

Elise Alméras, Olivier Masbernat, Frédéric Risso, Rodney O. Fox. Fluctuations in inertial dense homogeneous suspensions. Physical Review Fluids, 2019, 4 (10), pp.0. 10.1103/PhysRevFluids.4.102301 . hal-02318688

\section{HAL Id: hal-02318688 \\ https://hal.science/hal-02318688}

Submitted on 17 Oct 2019

HAL is a multi-disciplinary open access archive for the deposit and dissemination of scientific research documents, whether they are published or not. The documents may come from teaching and research institutions in France or abroad, or from public or private research centers.
L'archive ouverte pluridisciplinaire $\mathbf{H A L}$, est destinée au dépôt et à la diffusion de documents scientifiques de niveau recherche, publiés ou non, émanant des établissements d'enseignement et de recherche français ou étrangers, des laboratoires publics ou privés. 


\section{OATAO \\ Open Archive Toulouse Archive Ouverte}

\section{Open Archive Toulouse Archive Ouverte (OATAO)}

OATAO is an open access repository that collects the work of Toulouse researchers and makes it freely available over the web where possible

This is a Publisher's version published in: http://oatao.univ-toulouse.fr/24545

Official URL: https://doi.org/10.1103/PhysRevFluids.4.102301

\section{To cite this version:}

Alméras, Élise Rodney O. Fluctuations in inertial dense homogeneous suspensions. (2019) Physical Review Fluids, 4 (10). ISSN 2469-990X

Any correspondence concerning this service should be sent to the repository administrator: tech-oatao@listes-diff.inp-toulouse.fr 


\title{
Fluctuations in inertial dense homogeneous suspensions
}

\author{
Elise Alméras \\ FERMAT, Université de Toulouse, CNRS, INPT, INSA, UPS, Toulouse, France
}

\author{
Olivier Masbernat $\odot^{*}$ \\ Laboratoire de Génie Chimique, CNRS-INPT-UPS, Université de Toulouse, 31400 Toulouse, France
}

Frédéric Risso

Institut de Mécanique des Fluides de Toulouse (IMFT), Université de Toulouse, CNRS, Toulouse, France

Rodney O. Fox

Department of Chemical and Biological Engineering, Iowa State University, Ames, Iowa 50011-1098, USA

(Received 22 February 2019; published 16 October 2019)

\begin{abstract}
A theoretical model of liquid and particle random fluctuations is proposed for gravitydriven flows of inertial homogeneous suspensions. It is based on a paradigm assuming that fluctuations of both liquid velocity and particle slip velocity are driven by fluctuations of the phase indicator function. It is shown that this model accurately predicts the energy of the fluctuations of both the fluid and particle phases measured in a homogeneous solidliquid fluidized bed over a wide range of particle volume fractions, from $10 \%$ to $45 \%$.
\end{abstract}

DOI: 10.1103/PhysRevFluids.4.102301

Turbulent dispersed two-phase flows involving solid particles, liquid droplets, or bubbles randomly moving in an agitated fluid are ubiquitous in nature and industrial processes. Scaling agitation of both phases in these flows is a major issue since velocity fluctuations drive the mixing and interphase transfer processes of chemical species and heat. Two contrasting situations are encountered [1]. In the first [2], the agitation is dominated by the turbulence of the continuous phase and the fundamental mechanisms are (i) the dispersion of the particles by the turbulence that can be described by Tchen-Hinze theory [3], that is referred to as one-way coupling in numerical simulations, and (ii) the modulation of the turbulence by the particles, also known as two-way coupling [4]. In the second, the continuous-phase agitation is generated by the motion of the particles relative to the fluid, as is the case with gravity-driven dispersed flows such as liquid fluidized beds [5], settling suspensions [6,7], or bubbly flows [8].

This latter case is particularly complex since the fluctuations of the two phases result from each other and can therefore not be described separately. Indeed, random fluctuations in the liquid result from flow disturbances generated around particles, while particle fluctuations are caused by the fluid agitation. Moreover, the fluid agitation, usually referred to as pseudoturbulence, is of a different nature from shear-induced turbulence and involves a scaling that differs from Kolmogorov's theory.

The strong interplay between the statistics of motion of both phases is still challenging classical statistical modeling either based on a two-fluid model formulation [9-11] or the kinetic theory of granular flows $[12,13]$. Despite the increasing power of numerical tools, there is no reliable

*olivier.masbernat@ensiacet.fr 
law proposed thus far to relate continuous-phase and particle-phase agitation in pseudoturbulent suspension flows.

Introducing the fluctuations of the particle volume fraction, some theoretical developments $[14,15]$ suggest a strong influence of the variance of the fluctuations of the particle concentration upon the agitation of the particle phase (granular temperature). However, the definitions of volume fraction fluctuations, either based on the particle number density or on the ratio of solid to fluid volumes, rely on a finite control volume and thus depend on the ratio between this volume and that of a particle. Consequently, the concept of a local stochastic volume fraction needs to be treated carefully and can be a major source of confusion. Such a pitfall can be avoided by considering the fluctuations of the phase indicator function of the solid phase, which is the mathematically well-posed stochastic variable that naturally characterizes the local fluctuations of concentration. In this Rapid Communication, we develop a simple model for both liquid and particle agitation in gravity-driven suspension flows at relatively large inertia. It is based upon the correlation between the fluctuations of both the liquid and slip velocities and those of the phase indicator function, the average of which is equal to the volume fraction. This model reproduces with a very good accuracy experimental data collected in a liquid fluidized bed over a wide range of volume fractions.

We consider a homogeneous suspension of particles at a significant volume fraction, larger than $10 \%$. This corresponds to solid-liquid fluidized beds (also named liquid fluidized beds) or equivalently to settling particles in a fluid at rest, far from the walls. The particles move through the liquid under the action of gravity, so that the mean slip velocity of the particles relative to the fluid fixes the velocity scale of all motions within the suspension. In general, the fluctuations of the carrying phase induced by the dispersed phase include two contributions [16]: the disturbances generated in the vicinity of each particle and the turbulent fluctuations resulting from the instability of the flow through a random array of particles. At the large values of $\phi$ considered here, turbulence cannot develop in the confined interstitial region between the particles and local particle disturbances thus dominate.

In all what follows, brackets $\langle\cdot\rangle$ refer to the ensemble average. However, since we focus on flows that are homogeneous and stationary, we will assume that all stochastic processes are ergodic in space and time so that both time and spatial averages are equivalent to an ensemble average.

The mean slip velocity in such a flow is known to scale with $\phi$ according to the so-called Richardson-Zaki law [17]. Denoting $\left\langle U_{l z}\right\rangle$ the mean velocity of the liquid in the vertical direction and $\left\langle U_{p z}\right\rangle$ that of the particles, this law reads

$$
\left|\left\langle U_{l z}\right\rangle-\left\langle U_{p z}\right\rangle\right|=F(\phi)=U_{F 0}(1-\phi)^{n-1},
$$

where $U_{F 0}$ is a reference velocity slightly smaller (typically by $10 \%-20 \%$ ) than the terminal velocity $V_{t}$ of a single particle, and exponent $n$ is a function of the reference particulate Reynolds number, $\operatorname{Re}_{p 0}=\rho_{l} V_{t} d_{p} / \mu_{l}$, or Archimedes number, $A_{r}=\rho_{l}\left(\rho_{p}-\rho_{l}\right) g d_{p}^{3} / \mu_{l}^{2}$, with $\rho_{l}$ and $\mu_{l}$ being respectively the density and viscosity of the liquid phase, and $d_{p}$ and $\rho_{p}$ the particle diameter and density, respectively.

In Fig. 1, the average volume fraction $\phi$ is defined as the volume occupied by the particles divided by the considered volume of the suspension. It is also the average of the phase indicator function $\chi$, defined at any point $\boldsymbol{x}$ of the whole domain and at any time $t$ as follows,

$$
\begin{gathered}
\chi(x, t)=0 \text { when } \boldsymbol{x} \text { belongs to the liquid phase, } \\
\chi(\boldsymbol{x}, t)=1 \text { when } \boldsymbol{x} \text { belongs to the solid phase. }
\end{gathered}
$$

By definition, $\langle\chi(\boldsymbol{x}, t)\rangle=\phi$. Due to particle random motion in the domain, this function is random and possesses the following properties: $\chi^{2}=\chi,(1-\chi)^{2}=(1-\chi)$, and $\chi(1-\chi)=0$.

Agitation of the liquid phase in these flows results from the wakes downstream of the particles and can be simply deduced from the scheme of Fig. 1. The instantaneous vertical velocity at points located between particles (interstitial region) is distributed around the average liquid-phase velocity $\left\langle U_{l z}\right\rangle$, whereas that of the points located in the wake behind a particle is distributed around the 


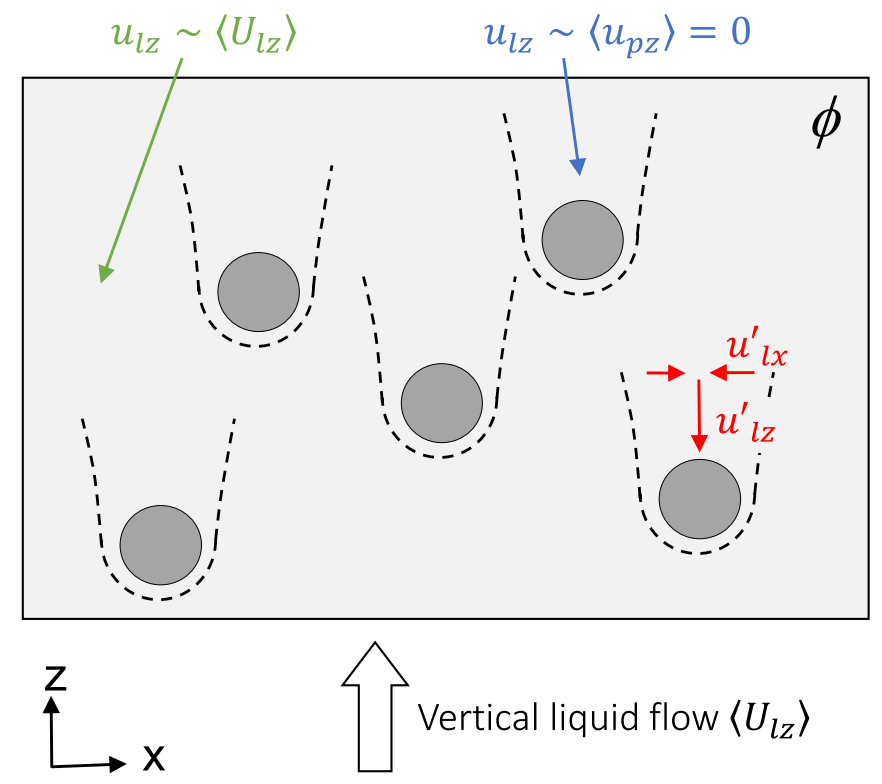

FIG. 1. Scheme of a gravity-driven suspension flow in the reference frame where $\left\langle U_{p z}\right\rangle=0$.

average particle velocity $\left\langle U_{p z}\right\rangle$, which is zero in the selected reference frame. It results that the main contribution to the liquid vertical velocity fluctuations at a position $\boldsymbol{x}$ of the domain, $u_{l z}^{\prime}=$ $u_{l z}-\left\langle U_{l z}\right\rangle$ is of the order $-\left\langle U_{l z}\right\rangle$ in the wake attached to a particle and conditioned by the occurrence of the presence of a particle in this region of the flow. Because the probability that a particle is close to a given location is proportional to $\phi$ [18], it is natural to consider that this occurrence is characterized by the fluctuations of the phase indicator function, $\chi^{\prime}=\chi-\phi$. This suggests the following form for $u_{l z}^{\prime}$,

$$
u_{l z}^{\prime}=-\gamma_{z}\left\langle U_{l z}\right\rangle \chi^{\prime}
$$

Since $\chi^{\prime}$ is a discrete variable that takes only the values $-\phi$ or $1-\phi$ and $u_{l z}^{\prime}$ is a continuous variable, $\gamma_{z}$ should be a continuous stochastic variable, which is statistically independent of $\chi^{\prime}$. In the aim to derive a simple model we will identify $\gamma_{z}$ to its average value, $\gamma_{z}=\left\langle\gamma_{z}\right\rangle$, which is positive and assumed to be independent on $\phi$.

The vertical fluid entrainment induced by velocity fluctuations $u_{l z}^{\prime}$ drags a horizontal flux of liquid as a result of local mass conservation. This generates horizontal velocity fluctuations $u_{l x}^{\prime}$, which scale as the vertical component $u_{l z}^{\prime}$, but with a smaller magnitude and an undetermined sign (see Fig. 1),

$$
u_{l x}^{\prime}=\gamma_{x}\left\langle U_{l z}\right\rangle \epsilon\left|\chi^{\prime}\right|
$$

in which $\gamma_{x}$ is a constant of the same order but smaller than $\gamma_{z}$ and $\epsilon$ is a stochastic variable independent of $\chi^{\prime}$, taking a value of +1 or -1 with equal probability.

It is important to stress that Eqs. (3) and (4) have not been derived from primary principles. They constitute a model based on the idea that the instantaneous liquid fluctuations are proportional to $\chi^{\prime}$, an assumption that we propose to validate by experiments that have been designed for that purpose. Equations (3) and (4) lead to the following model for the velocity $i$ th-component variance and the fluctuating liquid kinetic energy $E_{f}$,

$$
\left\langle u_{l i}^{\prime 2}\right\rangle=\gamma_{i}^{2}\left\langle U_{l z}\right\rangle^{2}\left\langle\chi^{\prime 2}\right\rangle, \quad E_{f}=\frac{1}{2} \sum_{i=1}^{3}\left\langle u_{l i}^{\prime 2}\right\rangle=\frac{1}{2}\left\langle U_{l z}\right\rangle^{2}\left\langle\chi^{\prime 2}\right\rangle \sum_{i=1}^{3} \gamma_{i}^{2} .
$$


A direct consequence of our assumptions is that the anisotropy ratio of the velocity components of the liquid phase should be independent of the dispersed phase volume fraction $\phi$.

The derivation of the variance of the phase indicator function in (5) is straightforward, using the definition and properties of $\chi$ [Eq. (2)],

$$
\left\langle\chi^{\prime 2}\right\rangle=\left\langle(\chi-\phi)^{2}\right\rangle=\langle\chi\rangle-\phi^{2}=\phi-\phi^{2}=\phi(1-\phi) .
$$

Equations (5) and (6) thus provide a general model for the variance of the liquid velocity fluctuations in gravity-driven inertial suspensions. Inserting relation (1) for the slip velocity into (5), one finds

$$
\left\langle u_{l i}^{\prime 2}\right\rangle=\gamma_{i}^{2}\left\langle U_{l z}\right\rangle^{2} \phi(1-\phi)=\gamma_{i}^{2} U_{F 0}^{2} \phi(1-\phi)^{2 n-1},
$$

where $\gamma_{i}$ is the constant scaling factor of the $i$ th component.

Particle-phase agitation results from a filtering effect of liquid-phase agitation, the amplitude of which is driven by particle inertia (or density ratio $\left.\rho_{p} / \rho_{l}\right)$. High inertia particles $\left(1 \ll \rho_{p} / \rho_{l}\right)$ weakly respond to liquid-phase agitation, whereas lower inertia particles $\left(\rho_{p} / \rho_{l} \leqslant 1\right)$ mimic fluid-particle random motion. The first zero-order model aimed at predicting this behavior is provided by TchenHinze theory, based on the resolution in spectral space of a single particle equation of motion in an undisturbed turbulent flow [11,19]. Assuming an exponential decay (in space and/or time) of liquid velocity fluctuations, this model predicts the fluctuating kinetic energy of the particle phase $E_{p}$ as a function of $E_{f}$,

$$
E_{p}=E_{f}\left(\frac{b^{2}+\eta_{f p}}{1+\eta_{f p}}\right),
$$

with $b=\frac{1+C_{A}}{\rho_{p} / \rho_{l}+C_{A}}$ and $C_{A}=1 / 2$ is the added-mass coefficient for a spherical particle.

In Eq. (8), the parameter $\eta_{f p}$ represents the ratio between two characteristic timescales: (i) the decorrelation time of liquid fluctuations following — or seen by — the particle (Lagrangian timescale) and (ii) the response time of the particle accounting for drag and added mass $[11,19]$. With the flow considered here, the fluctuations of the liquid phase result from localized disturbances in the vicinity of particles suggesting that this ratio is of the order of unity. Even if, by definition, $\eta_{f p}$ can be computed at any volume fraction $\phi$ in Eq. (8), the general solution of momentum conservation leading to scaling relations (8) [or (9)] does not account for the modulation effect by neighboring particles. Moreover, it can be shown that the limit of $\eta_{f p}$ when $\phi$ tends towards zero is zero, so Eq. (8) becomes

$$
\lim _{\phi \rightarrow 0} E_{p}=b^{2} E_{f}
$$

As a consequence, Eq. (9) must be taken as the asymptotic form of the particle filtering effect of liquid fluctuations in the limit of dilute systems.

Therefore we need next to establish a model for particle agitation accounting for the finitevolume-fraction effect on the same grounds as that derived for the liquid phase in Eqs. (3) and (4). For this purpose, we will consider that the relation between the fluctuations of the relative velocity between the fluid and the particles is similar to the relation between their average values. The average velocity between the two phases depends on the average volume fraction $\phi$ and thus $\left\langle U_{l z}\right\rangle-\left\langle U_{p z}\right\rangle=F(\phi)$. To apply this relation to the velocity fluctuations, we make use again of $\chi^{\prime}$ to characterize the fluctuations of concentration. Then, keeping only the first term of the expansion of $F$ in $\chi^{\prime}$ around the local phase volume fraction $\phi$, one comes to

$$
u_{l z}^{\prime}-u_{p z}^{\prime}=k_{z} \frac{d F}{d \phi} \chi^{\prime}
$$


Similarly to $\gamma_{z}$ in Eq. (4), the proportionality factor $k_{z}$ is taken equal to its average $\left\langle k_{z}\right\rangle>0 . F(\phi)$ is given by Eq. (1). Squaring Eq. (10) and averaging leads to

$$
\left\langle u_{l z}^{\prime 2}\right\rangle-2\left\langle u_{l z}^{\prime} u_{p z}^{\prime}\right\rangle+\left\langle u_{p z}^{\prime 2}\right\rangle=k_{z}^{2}\left(\frac{d F}{d \phi}\right)^{2}\left\langle\chi^{\prime 2}\right\rangle .
$$

In this equation, the second term on the left-hand side is the intercorrelation between the fluid and particle fluctuations. In terms of kinetic energy, it represents the amount of kinetic energy exchanged between the fluid and the particles. From Eqs. (3) and (10), this term can be easily expressed as a function of the liquid variance,

$$
\left\langle u_{l z}^{\prime} u_{p z}^{\prime}\right\rangle=\left\langle u_{l z}^{\prime 2}\right\rangle+k_{z} \frac{d F}{d \phi}\left\langle u_{l z}^{\prime 2}\right\rangle^{1 / 2}[\phi(1-\phi)]^{1 / 2} .
$$

Inserting (12) into (11) leads to the following relation between the particle and liquid velocity variances,

$$
\left\langle u_{p z}^{\prime 2}\right\rangle=\left\langle u_{l z}^{\prime 2}\right\rangle\left(1+\frac{k_{z}}{\left\langle u_{l z}^{\prime 2}\right\rangle^{1 / 2}} \frac{d F}{d \phi}[\phi(1-\phi)]^{1 / 2}\right)^{2} .
$$

As a result of the strong correlation between liquid horizontal and vertical fluctuating velocity components, we assume a similar form for the horizontal slip velocity fluctuations,

$$
\left\langle u_{p x}^{\prime 2}\right\rangle=\left\langle u_{l x}^{\prime 2}\right\rangle\left(1+\frac{k_{x}}{\left\langle u_{l x}^{\prime 2}\right\rangle^{1 / 2}} \frac{d F}{d \phi}[\phi(1-\phi)]^{1 / 2}\right)^{2},
$$

with $k_{x}>0$. Making use of fluidization law (1) and identity (2), Eqs. (13) and (14) provide an analytic relation between the velocity variances of particles and fluid, with a single parameter for each component, $k_{z}$ and $k_{x}$. Equation (7) provides the relations for the liquid velocity variance.

Note that the assumption of inertial regime does not seem to have been necessary so far for the development of the above equations, since the particle Reynolds (or Archimedes) number is only involved in the fluidization law, which is valid over a full range of Reynolds (or Archimedes) numbers. However, the particle Reynolds number needs to be large enough to ensure that the velocity disturbances generated by each particle vanish at a distance of the order of the particle diameter.

Although the model proposed above is quite simple, it is difficult to evaluate its accuracy against existing published experimental data, since these are scarce or incomplete. It is indeed required to have an accurate set of data for both phases' velocity distribution in a homogeneous suspension, and over a significant range of volume fraction. Therefore we have produced these data from a liquid fluidized bed experiment, following similar techniques as already developed by one of the authors in Ref. [5]. A 5-cm glass column is filled with an aqueous solution of glycerin and solid particles. Particles are fluidized by setting an upward flow of liquid. The height of the fluidized column gives the average particle volume fraction in the bed. Refractive indices of both phases are matched in order to allow the implementation of optical techniques, such as particle tracking or particle image velocimetry (PIV). Particle velocity distribution is measured by tracking colored particles in the bed with a high-speed video camera. Liquid velocity instantaneous two-dimensional field is measured by high-frequency PIV on a vertical laser sheet crossing the fluidized section. On each image, particles are masked and filtered out from the velocity signal. The particle and liquid velocity distributions are therefore obtained from these two independent techniques, and at each volume fraction (or equivalently fluidization velocity), their variances are fully converged. The system studied is composed of quasispherical Nafion ${ }^{\circledR}$ particles with a diameter $d_{p}=4 \mathrm{~mm}$ and a density of $\rho_{p}=1595 \mathrm{~kg} / \mathrm{m}^{3}$ fluidized by an aqueous solution of glycerine with a density $\rho_{f}=1045 \mathrm{~kg} / \mathrm{m}^{3}$ and a viscosity $\mu_{f}=1.76 \times 10^{-3} \mathrm{~Pa}$. The temperature of the fluidized bed is controlled and fixed at $19^{\circ} \mathrm{C}$. 


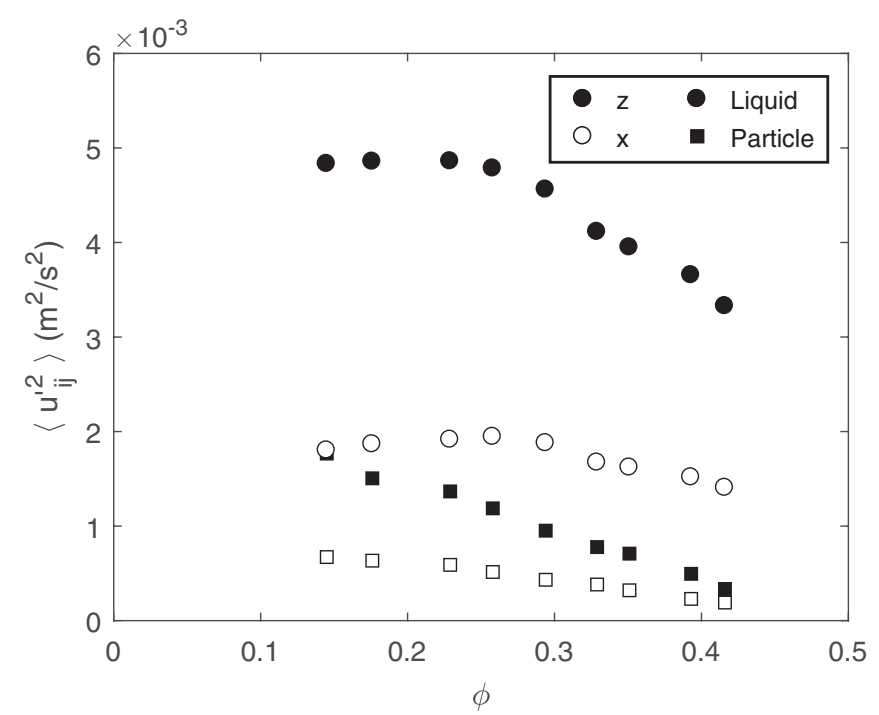

FIG. 2. Variance of fluctuating velocity components of liquid (circles) and particles (squares) as a function of volume fraction $\phi$ in the fluidized bed.

The fluidization law obtained for this system is consistent with the Richardson-Zaki's correlation [Eq. (1)] and gives a value of $U_{F 0}=0.189 \mathrm{~m} / \mathrm{s}$ close to the terminal velocity $V_{t}=0.223 \mathrm{~m} / \mathrm{s}$ and $n=2.31$. Because a single fluid-particle system is considered, the value of $\operatorname{Re}_{p 0}=530$ and $A_{r}=1.16 \times 10^{5}$ are fixed. However, the fluid-to-particle relative velocity changes with $\phi$ and the corresponding Reynolds number $\left(\operatorname{Re}_{p}=\rho_{l}\left\langle U_{l z}\right\rangle d_{p} / \mu_{l}\right)$ varies from 150 to 360. Evolution of the variance of the fluctuating velocity components of the liquid and particle is displayed as a function of volume fraction in Fig. 2. Particle agitation is significantly smaller than that of the fluid at each volume fraction, illustrating the filtering effect. Both tend to decay as the volume fraction increases in the investigated range. Liquid anisotropy seems to be weakly dependent on $\phi$. The model proposed for the liquid agitation implies that the velocity variance of each component divided by the mean slip (liquid) velocity should be proportional to the variance of the phase indicator function equal to $\phi(1-\phi)$.

This quantity has been reported in Fig. 3 for both components. The linear fit is remarkable over the entire range of volume fractions. On this graph, the anisotropic ratio $\gamma_{z} / \gamma_{x}$ is 1.56 . In order to evaluate the filtering effect given by Eq. (11), particle agitation has then been computed from Eqs. (13) and (14), using experimental values of the liquid-phase velocity variance for each volume fraction. Results (dashed line) are compared with experimental data (symbols) in Fig. 4. The quality of the prediction by Eqs. (13) and (14) with a single adjustable parameter $\left(k_{i}\right)$ is impressive, matching any slight slope change of the experimental trends.

Using Eqs. (5), (13), and (14), the fluctuating kinetic energy ratio between particles and liquid is plotted in Fig. 5. The model nicely fits the experimental data over the investigated range of solidphase fractions, between $10 \%$ and $45 \%$. Interestingly, there is no need in this model to require to a structure factor of a specific form, involving the value of volume fraction at maximum packing: The filtering effect of the liquid agitation by the particles is fully accounted by the fluidization law and the variance of the phase indicator function, which is an exact function of $\phi$. In the dilute limit, the model tends towards a smaller value $(0.41)$ than that predicted by Tchen-Hinze theory $(0.54)$. However, it is worth recalling that the model is not valid in this range.

We can conclude that this simple physical model based on the correlation between fluctuations of the velocities and the phase indicator function is relevant to predict liquid and particle agitation in any homogeneous buoyancy or gravity-driven suspension flow in the inertial regime $\left(\operatorname{Re}_{p}>100\right)$ for 


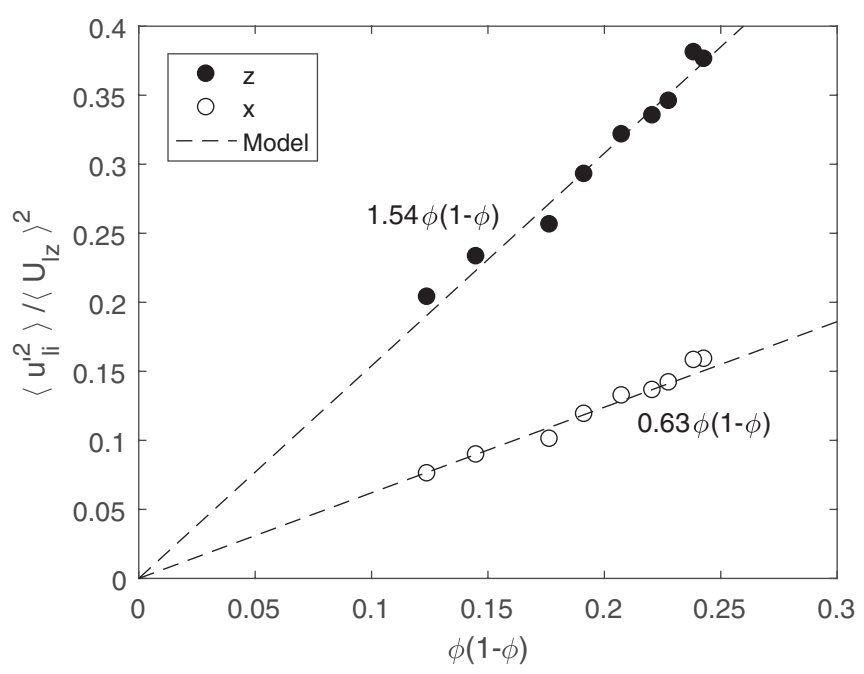

FIG. 3. Normalized variance of liquid fluctuating velocity component as a function of phase indicator function variance. Dashed line: Model based on Eq. (5).

moderate to high volume fractions $(\phi>0.1)$. This constitutes a significant improvement compared to current statistical models, either arising from two-fluid modeling or kinetic theory of granular media, and sheds light on the nature of pseudoturbulence in concentrated suspensions and in particular on the role of concentration fluctuations. Future work should focus on validating the model over different ranges of Reynolds number and density ratio. Furthermore, the modeling of diffusion mechanisms in such systems should be revisited in light of the proposed paradigm.

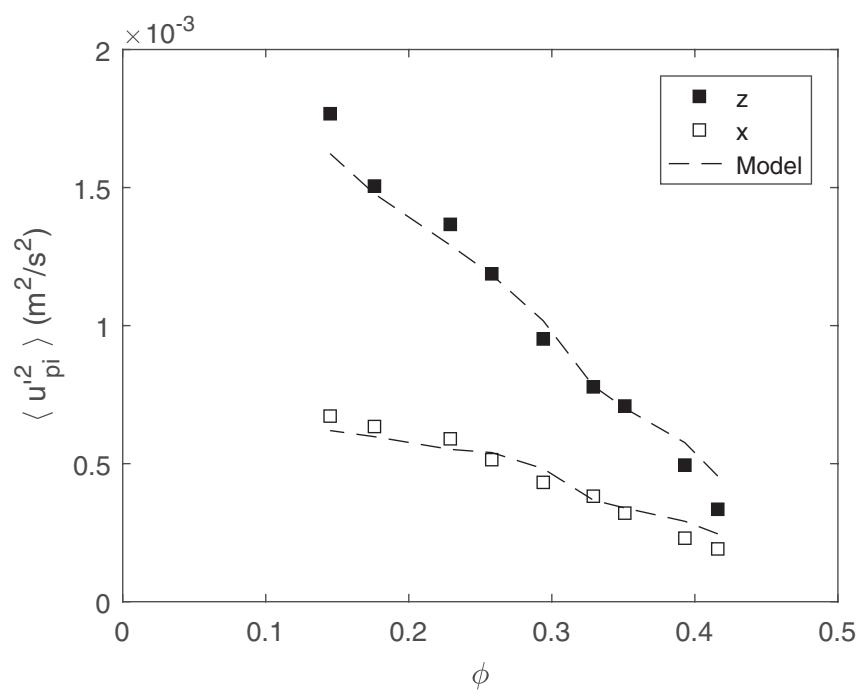

FIG. 4. Variance of particle fluctuating velocity components as a function of volume fraction. Black symbols: Vertical component. White symbols: Horizontal components. Dashed line: Model based on Eqs. (13) and (14), with $k_{z}=0.35$ and $k_{x}=0.21$. 


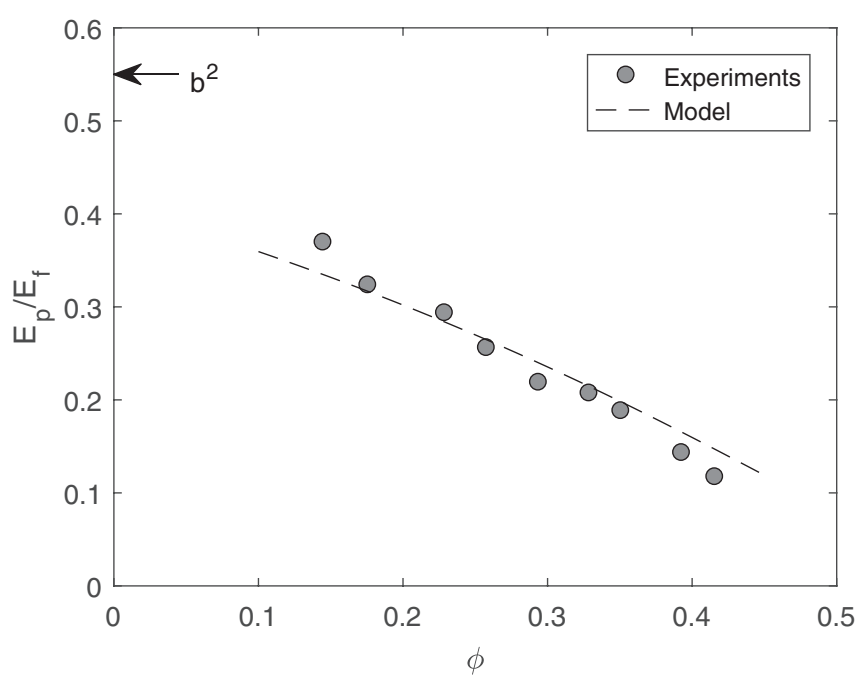

FIG. 5. Particle-to-liquid fluctuating kinetic energy ratio. $b^{2}=0.54$ for the present system is the limit of this ratio when $\phi \rightarrow 0$, as predicted by Tchen-Hinze theory.

This work has been carried out thanks to financial support from the research programm AAP 2015 of Idex UNITI - BIREM conv-ANR-11-Idex-0002-02. The authors acknowledge the MESR, Région Occitanie and Toulouse Métropole (CPER 2007-2013) and Emmanuel Cid (IR-CNRS, LGC) for assistance with the PIV measurements and data processing.

[1] F. Risso, Agitation, mixing, and transfers induced by bubbles, Annu. Rev. Fluid Mech. 50, 25 (2018).

[2] S. Balanchandar and J. Eaton, Turbulent dispersed multiphase flows, Annu. Rev. Fluid Mech. 42, 111 (2010).

[3] J. Abrahamson, Collision rates of small particles in a vigorously turbulent field, Chem. Eng. Sci. 30, 1371 (1975).

[4] P. Gualtieri, F. Battista, and C. M. Casciola, Turbulence modulation in heavy-loaded suspensions of tiny particles, Phys. Rev. Fluids 2, 034304 (2017).

[5] A. Aguilar-Corona, O. Masbernat, R. Zenit-Camacho, and B. Figueroa-Espinoza, in Experimental and Theoretical Advances in Fluid Dynamics, edited by J. Klapp (Springer, Berlin, 2012), pp. 39-51.

[6] X. Yin and D. Koch, Hindered settling velocity and microstructure in suspensions of solid spheres with moderate Reynolds numbers, Phys. Fluids 19, 093302 (2007).

[7] H. Nicolai, B. Herzhaft, E. Hinch, L. Oger, and E. Guazzelli, Particle velocity fluctuations and hydrodynamic self-diffusion of sedimenting non-Brownian spheres, Phys. Fluids 7, 12 (1995).

[8] G. Riboux, F. Risso, and D. Legendre, Experimental characterization of the agitation generated by bubbles rising at high Reynolds number, J. Fluid Mech. 643, 509 (2010).

[9] T. Anderson and R. Jackson, A fluid mechanical description of fluidized beds, Ind. Eng. Chem. Fundam. 6, 527 (1967).

[10] P. Duru, M. Nicolas, J. Hinch, and E. Guazzelli, Constitutive laws in liquid-fluidized beds, J. Fluid Mech. 452, 371 (2002).

[11] O. Simonin, E. Deutsch, and J. Minier, Eulerian prediction of the fluid/particle correlated motion in turbulent two-phase flows, Appl. Sci. Res. 51, 275 (1993).

[12] J. Ding and D. Gidaspow, A bubbling fluidization model using kinetic theory of granular flow, AIChE J. 36, 523 (1990). 
[13] F. Gevrin, O. Masbernat, and O. Simonin, Granular pressure and particle velocity fluctuations prediction in liquid fluidized beds, Chem. Eng. Sci. 63, 2450 (2008).

[14] Y. Buyevich, Particulate stresses in dense disperse flow, Ind. Eng. Chem. Res. 38, 731 (1999).

[15] P. Segre, F. Liu, P. Umbanhowar, and D. Weitz, An effective gravitational temperature for sedimentation, Nature (London) 409, 594 (2001).

[16] F. Risso, Physical interpretation of probability density functions of bubble-induced agitation, J. Fluid Mech. 809, 240 (2016).

[17] J. Richardson and W. Zaki, Sedimentation and fluidisation: Part I, Chem. Eng. Res. Des. 75, S82 (1997).

[18] E. J. Hinch, An averaged-equation approach to particle interactions in a fluid suspension, J. Fluid Mech. 83, 695 (1977).

[19] E. Deutsch and O. Simonin, Large eddy simulation applied to the modelling of particulate transport coefficients in turbulent two-phase flows, in Proceedings of the 8th Symposium on Turbulent Shear Flows (Pennsylvania State University, University Park, PA, 1991). 